DOI: $10.17516 / 1997-1397-2020-13-2-218-230$

УДК 517.9

\title{
Asymptotic Analysis of Retrial Queueing System M/M/1 with Impatient Customers, Collisions and Unreliable Server
}

\author{
Elena Yu. Danilyuk* \\ Svetlana P. Moiseeva ${ }^{\dagger}$ \\ National Research Tomsk State University \\ Tomsk, Russian Federation \\ Janos Sztrik ${ }^{\ddagger}$ \\ University of Debrecen \\ Debrecen, Hungary
}

Received 29.11.2019, received in revised form 04.12.2019, accepted 20.01.2020

\begin{abstract}
The retrial queueing system of $M / M / 1$ type with Poisson flow of arrivals, impatient customers, collisions and unreliable service device is considered in the paper. The novelty of our contribution is the inclusion of breakdowns and repairs of the service into our previous study to make the problem more realistic and hence more complicated. Retrial time of customers in the orbit, service time, impatience time of customers in the orbit, server lifetime (depending on whether it is idle or busy) and server recovery time are supposed to be exponentially distributed. An asymptotic analysis method is used to find the stationary distribution of the number of customers in the orbit. The heavy load of the system and long time patience of customers in the orbit are proposed as asymptotic conditions. Theorem about the Gaussian form of the asymptotic probability distribution of the number of customers in the orbit is formulated and proved. Numerical examples are given to show the accuracy and the area of feasibility of the proposed method.
\end{abstract}

Keywords: retrial queue, impatient customers, collisions, unreliable server, asymptotic analysis.

Citation: E.Yu.Danilyuk, S.P.Moiseeva, J.Sztrik, Asymptotic Analysis of Retrial Queueing System M/M/1 with Impatient Customers, Collisions and Unreliable Server, J. Sib. Fed. Univ. Math. Phys., 2020, 13(2), 218-230. DOI: 10.17516/1997-1397-2020-13-2-218-230.

The ever increasing volume of information for designing communication systems in an optimal way requires new methods and approaches. More and more business processes involve big data transmission under limited capacities of devices. Therefore, developing of appropriate mathematical models of modern telecommunication systems and modifying of existing ones are very important. Queueing systems with repeated calls, or retrial queueing systems are suitable models for telecommunication systems. They are characterized by the feature that an arriving customer finding the server busy does not join a queue and does not leave the system immediately, but goes to some virtual place (orbit), and then it tries to get service again after some random time. A comprehensive description and comparison of classical queueing systems of retrial queues can be found in books by J. Artalejo and A. Gómez-Corral [1], J. Artalejo and G. Falin [2], G. Falin and J. Templeton [3], just to mentions some of them.

\footnotetext{
*daniluc.elena.yu@gmail.comｈttps://orcid.org/0000-0002-7016-492X

${ }^{\dagger}$ smoiseeva@mail.ru htps://orcid.org/0000-0001-9285-1555

${ }^{\ddagger}$ sztrik.janos@inf.unideb.hu https://orcid.org/0000-0002-5303-818X

(C) Siberian Federal University. All rights reserved
} 
The present paper generalizes the results obtained in $[4,5]$. We find the asymptotic stationary distribution of the number of calls in the orbit for the system under consideration. Collisions in the model usually arise in the analysis of communication networks when another message is transmitted during the transmission of a previous message. Such messages collide. They are considered distorted and both go into the orbit from where they ask the device for servicing again after a random delay time, see for example [4-8].

Impatience of calls in the orbit is understood as the case when a customer in the orbit can leave the orbit after a random time without service [4,5,7,9-11]. But there is another way to specify impatience, for example, non-persistence, balking and reneging are used [8, 12-15]. Balking and reneging are fundamental concepts in queuing introduced by Anker, Gafarian [16], Haight [17] and Bareer [18]. They state that an arriving customer shows the least interest in joining a system which is already crowded. This behaviour is referred to as balking. Balking was applied to retention of reneged customers [19-23]. A comprehensive review of queueing systems with impatient customers can be found in [24].

In practice some components of the systems are subject to random breakdowns. Then it is very important to study reliability of retrial queues with server breakdowns and repairs because of the limited ability of repairs and heavy influence of the breakdowns on the performance of the system. Retrial queues with an unreliable server were studied, see for example $[5,14,25,26]$ and references therein.

More references on important papers devoted to the research of retrial queueing systems of various types (with impatient customers, collisions, and unreliable server) are given in our previous papers $[4,5,7,9-11,25,26]$.

The novelty of our contribution is the inclusion of breakdowns and repairs of the service into our previously developed models to make the problem more realistic and hence more complicated. In the present paper we continue to use an asymptotic analysis method developed at the Tomsk State University that is widely applied for the study of RQ -systems. This method makes it possible to obtain analytical result for different types of queueing systems and networks under specific asymptotic conditions.

The structure of the present work is as follows. Mathematical model of the novel retrial queueing system discussed in the paper and the problem statement are presented in Sect. 1. In Sect. 2 the detailed description of the model and the system of Kolmogorov equations for the stationary state probabilities are given. Sect. 3 consists of the solution of the problem by the asymptotic analysis method. Theorem on stationary probability distribution of the number in the orbit for retrial queueing system of $\mathrm{M} / \mathrm{M} / 1$ type with impatient calls in the orbit, collisions and unreliable server under a long delay of calls in orbit and long time patience of calls in the orbit condition is formulated and proved in this section. Sect. 4 deals with some numerical examples that prove the theoretical results and illustrate the applicability of the proposed approach. Sect. 5 concludes the paper.

\section{Description of the mathematical model}

We consider a single server RQ-system with Poisson arrival process with parameter $\lambda$ for the primary calls. A customer that finds the server idle takes it for service for an exponentially distributed random time with parameter $\mu$. If the server is busy an arriving customer (either from the source or from the orbit) enters into a "collision" and both go into orbit. In the orbit each customer, called secondary calls, independently of others waits for a random time. 
The waiting time is exponentially distributed with parameter $\sigma$. If the server is busy again the request tries to occupy the device to obtain servicing as soon as possible. If the server is idle the secondary customer occupies it for service for an exponentially distributed random time with parameter $\mu$, that is, no difference between the service of primary and secondary calls.

We assume that server is unreliable, that is, the lifetime is supposed to be exponentially distributed with rate $\gamma_{0}$ if the server is idle and with parameter $\gamma_{1}$ if it is busy. When the server breaks down it is immediately sent for repair and the recovery time is assumed to be exponentially distributed with rate $\gamma_{2}$. When the server is down the primary sources continue generation of customers and send them to the server. Similarly, customers may retry from the orbit to the server but all arriving customers immediately go into the orbit. Furthermore, in this unreliable model we suppose that interrupted request goes to the orbit immediately and it's next service is independent of the interrupted one. All random variables involved in the model construction are assumed to be independent of each other.

Moreover, a customer in the orbit leaves the system without service after a random time which has an exponential distribution with rate $\alpha$, demonstrating the "impatience" property. Fig. 1 shows the model of the RQ-system $M / M / 1$ with impatient customers, collisions and unreliable server.

Our aims is to find the stationary distribution of the number of customers in the orbit for the described system.

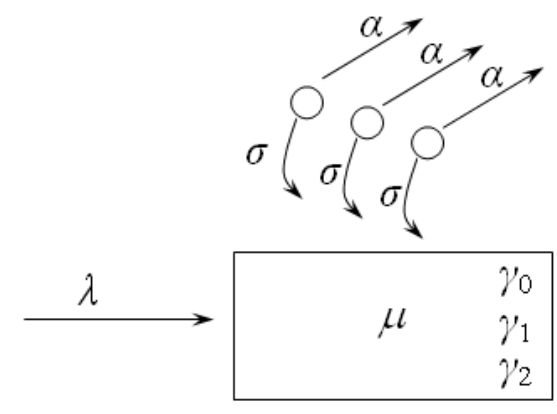

Fig. 1. Retrial queue $\mathrm{M} / \mathrm{M} / 1$ with impatient customers in the orbit, collisions and unreliable server

\section{System of the Kolmogorov differential equations}

Let us consider the Markov process $\{k(t), i(t)\}$ of changing the states of the RQ-system under consideration, where $i(t)$ is the number of customers in the orbit at time $t, i(t)=0,1,2, \ldots$ and $k(t)$ defines the state of the server at time $t$ and takes one of three values:

$$
k(t)= \begin{cases}0, & \text { if the device is idle, } \\ 1, & \text { if the device is busy, } \\ 2, & \text { if the device is down (under repair) }\end{cases}
$$

The joint probability that device is in state $k$ at time $t$ and $i$ customers are in orbit is denoted by $P\{k(t)=k, i(t)=i\}=P(k, i, t)$.

To obtain the probability distribution $P\{k(t)=k, i(t)=i\}=P(k, i, t)$ for the states of the 
considered RQ-system we derive a system of the Kolmogorov differential equations

$$
\left\{\begin{aligned}
\frac{\partial P(0, i, t)}{\partial t} & =-\left(\lambda+i \sigma+i \alpha+\gamma_{0}\right) P(0, i, t)+(i+1) \alpha P(0, i+1, t)+ \\
& +(i-1) \sigma P(1, i-1, t)+\mu P(1, i, t)+\lambda P(1, i-2, t)+\gamma_{2} P(2, i, t) \\
\frac{\partial P(1, i, t)}{\partial t} & =-\left(\lambda+\mu+i \sigma+i \alpha+\gamma_{1}\right) P(1, i, t)+\lambda P(0, i, t)+ \\
& +(i+1) \sigma P(0, i+1, t)+(i+1) \alpha P(1, i+1, t) \\
\frac{\partial P(2, i, t)}{\partial t} & =-\left(\lambda+i \alpha+\gamma_{2}\right) P(2, i, t)+\lambda P(2, i-1, t)+ \\
& +(i+1) \alpha P(2, i+1, t)+\gamma_{0} P(0, i, t)+\gamma_{1} P(1, i-1, t)
\end{aligned}\right.
$$

$i=0,1,2, \ldots$

Since customers are "impatient" the considered system has a stationary distribution for any values of $\lambda$ and $\mu$. Let $\lim _{t \rightarrow \infty} P(k, i, t)=P(k, i)=P_{k}(i), k=\{0 ; 1 ; 2\}$. Then we can write system (1) in the following form

$$
\left\{\begin{array}{l}
-\left(\lambda+i \sigma+i \alpha+\gamma_{0}\right) P_{0}(i)+(i+1) \alpha P_{0}(i+1)+(i-1) \sigma P_{1}(i-1)+\mu P_{1}(i)+ \\
\quad+\lambda P_{1}(i-2)+\gamma_{2} P_{2}(i)=0 \\
-\left(\lambda+\mu+i \sigma+i \alpha+\gamma_{1}\right) P_{1}(i)+\lambda P_{0}(i)+(i+1) \sigma P_{0}(i+1)+(i+1) \alpha P_{1}(i+1)=0 \\
-\left(\lambda+i \alpha+\gamma_{2}\right) P_{2}(i)+\lambda P_{2}(i-1)+(i+1) \alpha P_{2}(i+1)+\gamma_{0} P_{0}(i)+\gamma_{1} P_{1}(i-1)=0 \\
\sum_{i=0}^{\infty}\left(P_{0}(i)+P_{1}(i)+P_{2}(i)\right)=1
\end{array}\right.
$$

$i=0,1,2, \ldots$.

System (2) is a system of difference equations of infinite dimension with variable coefficients. Such system is very difficult to solve. Therefore, to get solution we propose two approaches: asymptotic and numerical ones.

The numerical algorithm for finding the stationary probabilities is based on the reduction of dimension of system (2). To do that we represent system (2) for $i=0,1,2, \ldots, N$ as

$$
P S=B
$$

where the row vector $P$ of dimension $3(N+1)$ is the desired stationary probability distribution of the number of customers in the orbit for each state of the device $k=\{0,1,2\}$

$$
P=(P(0) P(1) P(2))
$$

and $P(0), P(1), P(2)$ are row vectors with elements $P(0, i), P(1, i), P(2, i), i=0,1,2, \ldots, N$, respectively. Matrix $S$ of dimension $3(N+1) \times(3(N+1)+1)$ is represented in the block form as

$$
S=\left(\begin{array}{cccc}
S_{11} & S_{12} & S_{13} & S_{14} \\
S_{21} & S_{22} & S_{23} & S_{24} \\
S_{31} & S_{32} & S_{33} & S_{34}
\end{array}\right)
$$

where $S_{11}=\left\|s_{i j}^{11}\right\|_{1}^{N+1}, S_{12}=\left\|s_{i j}^{12}\right\|_{1}^{N+1}, S_{13}=\left\|s_{i j}^{13}\right\|_{1}^{N+1}, S_{21}=\left\|s_{i j}^{21}\right\|_{1}^{N+1}, S_{22}=\left\|s_{i j}^{22}\right\|_{1}^{N+1}$, $S_{23}=\left\|s_{i j}^{23}\right\|_{1}^{N+1}, S_{31}=\left\|s_{i j}^{31}\right\|_{1}^{N+1}, S_{32}=\left\|s_{i j}^{32}\right\|_{1}^{N+1}, S_{33}=\left\|s_{i j}^{33}\right\|_{1}^{N+1}$ are sparse matrices with 
non-zero elements defined as

$$
\begin{gathered}
s_{i i}^{11}=-\lambda-(i-1)(\sigma+\alpha)-\gamma_{0}, \quad s_{i+1, i}^{11}=i \alpha, \\
s_{i i}^{21}=\mu, \quad s_{i, i+1}^{21}=(i-1) \sigma, \quad s_{i, i+2}^{21}=\lambda, \\
s_{i i}^{31}=\gamma_{2}, \\
s_{i i}^{12}=\lambda, \quad s_{i, i-1}^{12}=i \sigma, \\
s_{i i}^{22}=-\left(\lambda+\mu+\gamma_{1}+(i-1)(\sigma+\alpha)\right), \quad s_{i+1, i}^{22}=i \alpha, \\
s_{i i}^{13}=\gamma_{0}, \\
s_{i, i+1}^{23}=\gamma_{1}, \\
s_{i i}^{33}=-\left(\lambda+\gamma_{2}+(i-1) \alpha\right), \quad s_{i+1, i}^{33}=i \alpha, \quad s_{i, i+1}^{33}=\lambda .
\end{gathered}
$$

Blocks $S_{14}, S_{24}, S_{34}$ are unit vector columns of dimension $(N+1)$, row vector $B=\left\|b_{i}\right\|$ of dimension $3(N+1)+1$ is a row of free coefficients with elements $b_{n}=0(n=0,1,2, \ldots, N-1)$, $b_{N}=1$.

We solve (3) with the use of the Mathcad software package. We choose $N$ to be so large that probabilities $P(0, N), P(1, N), P(2, N)$ are equal to the machine zero.

The numerical algorithm provides satisfactory accuracy but it has a drawback due to the limited computational capacity of the computer. Therefore, analytical methods are needed to calculate the probability distribution of the number of customers in orbit for the considered RQ-system with impatient customers, collisions and unreliable server. They allow us to find a distribution for a system of any dimension. Thus the alternative to the numerical method is the method of asymptotic analysis.

\section{Asymptotic analysis}

To find the solution of system of equations (2) we propose another approach by using the method of asymptotic analysis under the assumption that there is a long delay between customers from the orbit and high "patience" customers, i.e., when $\sigma \rightarrow 0, \alpha \rightarrow 0$. We summarize the results of our study in the next Theorem 3.1.

Theorem 3.1. The stationary distribution of the number of customers in the orbit in the $R Q$ system $M / M / 1$ with impatient customers, collisions and unreliable server under the above assumptions and conditions $\alpha=q \sigma, q>0$ is the asymptotically normal distribution with mean $G_{1} / \sigma$ and variance $G_{2} / \sigma$. Here

$$
\begin{gathered}
G_{1}=\frac{\lambda-\mu R_{1}}{q}, \\
G_{2}=\frac{G_{1}\left(q+R_{0}\right)+\gamma_{1} f_{0}+\lambda R_{1}}{R_{0}-R_{1}+q}, \\
f_{1}=\frac{\left(2 \lambda+(1-q) G_{1}\right) R_{1}-(1+q) G_{1} R_{0}}{\gamma_{0}+\gamma_{1}+2 \gamma_{2}} .
\end{gathered}
$$

$R_{1}$ is the probability that the server is busy in the stationary regime of system operation. It is determined by equation

$$
\left(\gamma_{0}+\gamma_{1}+2 \gamma_{2}\right) \mu R_{1}^{2}-c R_{1}+\lambda(1+q) \gamma_{2}=0, \quad R_{1} \in[0 ; 1],
$$




$$
c=(1+q) \lambda\left(\gamma_{0}+\gamma_{1}+2 \gamma_{2}\right)+q\left(\gamma_{0}+\gamma_{2}\right)\left(\mu+\gamma_{1}\right)+\mu \gamma_{2}
$$

$R_{0}$ is the probability that the server is idle in the stationary regime of system operation. It is determined by equation

$$
R_{0}=\frac{\gamma_{2}-\left(\gamma_{1}+\gamma_{2}\right) R_{1}}{\gamma_{0}+\gamma_{2}}, \quad R_{0} \in[0 ; 1]
$$

Proof. The method of asymptotic analysis in queueing theory is the method of study of the equations to determine some characteristics of an queueing system under some limit (asymptotic) condition which is specific for any model and problem under consideration.

We introduce the partial characteristic functions as follows

$$
H_{k}(u)=\sum_{i=0}^{\infty} e^{j u i} P_{k}(i), \quad H_{k}(0)=\sum_{i=0}^{\infty} P_{k}(i) \triangleq R_{k},
$$

where $j=\sqrt{-1}, k=\{0,1,2\}$, and $R_{k}$ are stationary state probabilities of the process $k(t)$. It is obvious that $H(u)=H_{0}(u)+H_{1}(u)+H_{2}(u)$. follows

Using (9) and $H_{k}^{\prime}(u)=\frac{d H_{k}(u)}{d u}=j \sum_{i=0}^{\infty} i e^{j u i} P_{k}(i), k=\{0,1,2\}$, we can write system (2) as

$$
\left\{\begin{array}{l}
-\left(\lambda+\gamma_{0}\right) H_{0}(u)+j(\sigma+\alpha) H_{0}^{\prime}(u)+\mu H_{1}(u)-j \alpha e^{-j u} H_{0}^{\prime}(u)-j \sigma e^{j u} H_{1}^{\prime}(u)+ \\
\quad+\lambda e^{2 j u} H_{1}(u)+\gamma_{2} H_{2}(u)=0, \\
-\left(\lambda+\mu+\gamma_{1}\right) H_{1}(u)+j(\sigma+\alpha) H_{1}^{\prime}(u)+\lambda H_{0}(u)-j \alpha e^{-j u} H_{1}^{\prime}(u)-j \sigma e^{-j u} H_{0}^{\prime}(u)=0, \\
-\left(\lambda+\gamma_{2}\right) H_{2}(u)+j \alpha H_{2}^{\prime}(u)+\lambda e^{j u} H_{2}(u)-j \alpha e^{-j u} H_{2}^{\prime}(u)+\gamma_{0} H_{0}(u)+\gamma_{1} e^{j u} H_{1}(u)=0 .
\end{array}\right.
$$

Adding the first and the second equations by the third one of (10) we get the system below

$$
\left\{\begin{array}{l}
-\left(\lambda+\gamma_{0}\right) H_{0}(u)+\left(\mu+\lambda e^{2 j u}\right) H_{1}(u)+\gamma_{2} H_{2}(u)+j\left(\sigma+\alpha-\alpha e^{-j u}\right) H_{0}^{\prime}(u)- \\
\quad-j \sigma e^{j u} H_{1}^{\prime}(u)=0, \\
\lambda H_{0}(u)-\left(\lambda+\mu+\gamma_{1}\right) H_{1}(u)-j \sigma e^{-j u} H_{0}^{\prime}(u)+j\left(\sigma+\alpha-\alpha e^{-j u}\right) H_{1}^{\prime}(u)=0, \\
\gamma_{0} H_{0}(u)+\gamma_{1} e^{j u} H_{1}(u)-\left(\lambda+\gamma_{2}-\lambda e^{j u}\right) H_{2}(u)+j \alpha\left(1-e^{-j u}\right) H_{2}^{\prime}(u)=0, \\
{\left[\lambda\left(e^{j u}+1\right)+\gamma_{1}\right] H_{1}(u)+\lambda H_{2}(u)+j e^{-j u}(\sigma+\alpha) H_{0}^{\prime}(u)+j\left(\alpha e^{-j u}-\sigma\right) H_{1}^{\prime}(u)+} \\
\quad+j \alpha e^{-j u} H_{2}^{\prime}(u)=0 .
\end{array}\right.
$$

System (11) is the basic system for further analysis of retrial queueing system of $M / M / 1$ type with impatient customers in the orbit, collisions and unreliable server under a long delay of customers in orbit $(\sigma \rightarrow 0)$ and long time patience of customers in the orbit $(\alpha \rightarrow 0)$ conditions.

The proof of Theorem 3.1 is carried out in two stages.

Stage 1. Finding the first-order asymptotic.

Let us make the substitutions $\sigma=\varepsilon, \alpha=q \varepsilon, u=\varepsilon w, H_{k}(u)==F_{k}(w, \varepsilon), k=\{0,1,2\}$ in basic system (11), where $\varepsilon \rightarrow 0$. 
Since $H_{k}^{\prime}(u)=\frac{1}{\varepsilon} \frac{\partial F_{k}(w, \varepsilon)}{\partial w}, k=\{0,1,2\}$ systems of equations (11) can be written as

$$
\left\{\begin{array}{l}
-\left(\lambda+\gamma_{0}\right) F_{0}(w, \varepsilon)+\left(\mu+\lambda e^{2 j w \varepsilon}\right) F_{1}(w, \varepsilon)+\gamma_{2} F_{2}(w, \varepsilon)+ \\
\quad+j\left(1+q-q e^{-j w \varepsilon}\right) \frac{\partial F_{0}(w, \varepsilon)}{\partial w}-j e^{j w \varepsilon} \frac{\partial F_{1}(w, \varepsilon)}{\partial w}=0, \\
\lambda F_{0}(w, \varepsilon)-\left(\lambda+\mu+\gamma_{1}\right) F_{1}(w, \varepsilon)-j e^{-j w \varepsilon} \frac{\partial F_{0}(w, \varepsilon)}{\partial w}+j\left(1+q-q e^{-j w \varepsilon}\right) \frac{\partial F_{1}(w, \varepsilon)}{\partial w}=0, \\
\gamma_{0} F_{0}(w, \varepsilon)+\gamma_{1} e^{j w \varepsilon} F_{1}(w, \varepsilon)-\left(\lambda+\gamma_{2}-\lambda e^{j w \varepsilon}\right) F_{2}(w, \varepsilon)+j q\left(1-e^{-j w \varepsilon}\right) \frac{\partial F_{2}(w, \varepsilon)}{\partial w}=0, \\
\quad\left[\lambda\left(e^{j w \varepsilon}+1\right)+\gamma_{1}\right] F_{1}(w, \varepsilon)+\lambda F_{2}(w, \varepsilon)+j e^{-j w \varepsilon}(1+q) \frac{\partial F_{0}(w, \varepsilon)}{\partial w}+ \\
\quad+j\left(q e^{-j w \varepsilon}-1\right) \frac{\partial F_{1}(w, \varepsilon)}{\partial w}+j q e^{-j w \varepsilon} \frac{\partial F_{2}(w, \varepsilon)}{\partial w}=0 .
\end{array}\right.
$$

The transformation of equations (12) under $\varepsilon \rightarrow 0$ with $F_{k}(w)=\lim _{\varepsilon \rightarrow 0} F_{k}(w, \varepsilon), F_{k}^{\prime}(w)=$ $=\frac{d F_{k}(w)}{d w}, k=\{0,1,2\}$ leads to

$$
\left\{\begin{array}{l}
-\left(\lambda+\gamma_{0}\right) F_{0}(w)+(\mu+\lambda) F_{1}(w)+\gamma_{2} F_{2}(w)+j F_{0}^{\prime}(w)-j F_{1}^{\prime}(w)=0 \\
\lambda F_{0}(w)-\left(\lambda+\mu+\gamma_{1}\right) F_{1}(w)-j F_{0}^{\prime}(w)+j F_{1}^{\prime}(w)=0 \\
\gamma_{0} F_{0}(w)+\gamma_{1} F_{1}(w)-\gamma_{2} F_{2}(w)=0 \\
\lambda\left[F_{0}(w)+F_{1}(w)+F_{2}(w)\right]-\mu F_{1}(w)+j q\left[F_{0}^{\prime}(w)+F_{1}^{\prime}(w)+F_{2}^{\prime}(w)\right]=0
\end{array}\right.
$$

We seek solution of equation (13) $F_{k}(w), k=\{0,1,2\}$ in the form

$$
F_{k}(w)=R_{k} \Phi(w), \quad k=\{0,1,2\},
$$

where $R_{0}, R_{1}, R_{2}$ are defined in (9), $R_{0}+R_{1}+R_{2}=1, R_{k}=H_{k}(0)=F_{k}(0), k=\{0,1,2\}$, and $\Phi(w)$ is an unknown function.

Substituting (14) into (13), we obtain the system of differential equations with respect to function $\Phi(w)$

$$
\left\{\begin{array}{l}
{\left[-\left(\lambda+\gamma_{0}\right) R_{0}+(\mu+\lambda) R_{1}+\gamma_{2} R_{2}\right] \Phi(w)=j\left(R_{1}-R_{0}\right) \frac{d \Phi(w)}{d w}} \\
{\left[\lambda R_{0}-\left(\lambda+\mu+\gamma_{1}\right) R_{1}\right] \Phi(w)=j\left(R_{1}-R_{0}\right) \frac{d \Phi(w)}{d w}} \\
\gamma_{0} R_{0} \Phi(w)+\gamma_{1} R_{1} \Phi(w)-\gamma_{2} R_{2} \Phi(w)=0 \\
{\left[\lambda\left(R_{0}+R_{1}+R_{2}\right)-\mu R_{1}\right] \Phi(w)=-j q\left[R_{0}+R_{1}+R_{2}\right] \frac{d \Phi(w)}{d w}}
\end{array}\right.
$$

According to equations (15), we can find

$$
\Phi(w)=\exp \left\{G_{1} j w\right\}
$$

where $G_{1}$ is given in (4). It follows from the forth equation (15).

It is obvious that solution of system (15) exists when the following equalities are satisfied

$$
\left\{\begin{array}{l}
\left(\lambda+\gamma_{0}\right) R_{0}-(\mu+\lambda) R_{1}-\gamma_{2} R_{2}=\lambda R_{0}-\left(\lambda+\mu+\gamma_{1}\right) R_{1}, \\
\gamma_{0} R_{0}+\gamma_{1} R_{1}-\gamma_{2} R_{2}=0 \\
R_{0}+R_{1}+R_{2}=1 .
\end{array}\right.
$$


Expressions for $R_{0}, R_{1}, R_{2} \in[0 ; 1]$ can be obtained from system of equations (17). They are

$$
R_{0}=\frac{\gamma_{2}-\left(\gamma_{1}+\gamma_{2}\right) R_{1}}{\gamma_{0}+\gamma_{1}}, \quad R_{2}=\frac{\gamma_{0}-\left(\gamma_{0}-\gamma_{1}\right) R_{1}}{\gamma_{0}+\gamma_{2}}
$$

and $R_{1}$ is the root of equation

$$
\begin{gathered}
\left(\gamma_{0}+\gamma_{1}+2 \gamma_{2}\right) \mu R_{1}^{2}-c R_{1}+\lambda(1+q) \gamma_{2}=0 \\
c=(1+q) \lambda\left(\gamma_{0}+\gamma_{1}+2 \gamma_{2}\right)+q\left(\gamma_{0}+\gamma_{2}\right)\left(\mu+\gamma_{1}\right)+\mu \gamma_{2} .
\end{gathered}
$$

Equation (19) has at least one root $R_{1} \in[0 ; 1]$, and the proof of existence of $R_{1}$ is similar to that in [4].

Using (14), (16) and $\varepsilon=\sigma$ we can write the expression for the partial characteristic functions as follows

$$
H_{k}(u)=F_{k}(w, \varepsilon)=F_{k}(w)+o(\varepsilon) \approx F_{k}(w)=F_{k}\left(\frac{u}{\varepsilon}\right)=R_{k} \exp \left\{\frac{G_{1}}{\sigma} j u\right\},
$$

$k=\{0,1,2\}$, and $R_{0}, R_{1}, R_{2} \in[0 ; 1]$ are defined in (18), (19).

Using (20) and $R_{0}+R_{1}+R_{2}=1$ the pre-limit characteristic function $H(u)=H_{0}(u)+H_{1}(u)+$ $+H_{2}(u)$ under the assumption of a long delay of customers in the orbit and their high "patience" can be approximated by function $h_{1}(u)$

$$
h_{1}(u)=\exp \left\{\frac{G_{1}}{\sigma} j u\right\}
$$

which is the first-order asymptotic characteristic function (or the first-order asymptotic).

Stage 2. Finding the second-order asymptotic.

Taking into account (21), we assume

$$
H_{k}(u)=\exp \left\{\frac{G_{1}}{\sigma} j u\right\} H_{k}^{(2)}(u), \quad k=\{0,1,2\},
$$

in basic system of equations (11). Then systems of equations (11) can be rewritten as follows

$$
\left\{\begin{array}{l}
-\left[\lambda+\gamma_{0}+\frac{G_{1}}{\sigma}\left(\sigma+\alpha-\alpha e^{-j u}\right)\right] H_{0}^{(2)}(u)+\left(\mu+\lambda e^{2 j u}+G_{1} e^{j u}\right) H_{1}^{(2)}(u)+ \\
\quad+\gamma_{2} H_{2}^{(2)}(u)+j\left(\sigma+\alpha-\alpha e^{-j u}\right) \frac{d H_{0}^{(2)}(u)}{d u}-j \sigma e^{j u} \frac{d H_{1}^{(2)}(u)}{d u}=0 \\
\left(\lambda+G_{1} e^{-j u}\right) H_{0}^{(2)}(u)-\left[\lambda+\mu+\gamma_{1}+\frac{G_{1}}{\sigma}\left(\sigma+\alpha-\alpha e^{-j u}\right)\right] H_{1}^{(2)}(u)- \\
\quad-j \sigma e^{-j u} \frac{d H_{0}^{(2)}(u)}{d u}+j\left(\sigma+\alpha-\alpha e^{-j u}\right) \frac{d H_{1}^{(2)}(u)}{d u}=0, \\
\gamma_{0} H_{0}^{(2)}(u)+\gamma_{1} e^{j u} H_{1}^{(2)}(u)-\left[\lambda+\gamma_{2}-\lambda e^{j u}+\frac{G_{1} \alpha}{\sigma}\left(1-e^{-j u}\right)\right] H_{2}^{(2)}(u)+ \\
\quad+j \alpha\left(1-e^{-j u}\right) \frac{d H_{2}^{(2)}(u)}{d u}=0, \\
-e^{-j u} \frac{G_{1}}{\sigma}(\sigma+\alpha) H_{0}^{(2)}(u)+\left[\lambda\left(e^{j u}+1\right)+\gamma_{1}-\frac{G_{1}}{\sigma}\left(\alpha e^{-j u}-\sigma\right)\right] H_{1}^{(2)}(u)+\left(\lambda-\frac{G_{1} \alpha}{\sigma} e^{-j u}\right) \times \\
\quad \times H_{2}^{(2)}(u)+j e^{-j u}(\sigma+\alpha) \frac{d H_{0}^{(2)}(u)}{d u}+j\left(\alpha e^{-j u}-\sigma\right) \frac{d H_{1}^{(2)}(u)}{d u}+j \alpha e^{-j u} \frac{d H_{2}^{(2)}(u)}{d u}=0
\end{array}\right.
$$


Let $\sigma=\varepsilon^{2}, \alpha=q \varepsilon^{2}, u=\varepsilon w, H_{k}^{(2)}(u)=F_{k}^{(2)}(w, \varepsilon), k=\{0,1,2\}$, where $\varepsilon \rightarrow 0$. Then system (23) after some transformations becomes

$$
\left\{\begin{array}{l}
-\left[\lambda+\gamma_{0}+G_{1}\left(1+q-q e^{-j w \varepsilon}\right)\right] F_{0}^{(2)}(w, \varepsilon)+\left(\mu+\lambda e^{2 j w \varepsilon}+G_{1} e^{j w \varepsilon}\right) F_{1}^{(2)}(w, \varepsilon)+ \\
\quad+\gamma_{2} F_{2}^{(2)}(w, \varepsilon)+j \varepsilon\left(1+q-q e^{-j w \varepsilon}\right) \frac{\partial F_{0}^{(2)}(w, \varepsilon)}{\partial w}-j \varepsilon e^{j w \varepsilon} \frac{\partial F_{1}^{(2)}(w, \varepsilon)}{\partial w}=0 \\
\left(\lambda+G_{1} e^{-j w \varepsilon}\right) F_{0}^{(2)}(w, \varepsilon)-\left[\lambda+\mu+\gamma_{1}+G_{1}\left(1+q-q e^{-j w \varepsilon}\right)\right] F_{1}^{(2)}(w, \varepsilon)- \\
\quad-j \varepsilon e^{-j w \varepsilon} \frac{\partial F_{0}^{(2)}(w, \varepsilon)}{\partial w}+j \varepsilon\left(1+q-q e^{-j w \varepsilon}\right) \frac{\partial F_{1}^{(2)}(w, \varepsilon)}{\partial w}=0 \\
\gamma_{0} F_{0}^{(2)}(w, \varepsilon)+\gamma_{1} e^{j w \varepsilon} F_{1}^{(2)}(w, \varepsilon)-\left[\lambda+\gamma_{2}-\lambda e^{j w \varepsilon}+G_{1} q\left(1-e^{-j w \varepsilon}\right)\right] F_{2}^{(2)}(w, \varepsilon)+ \\
\quad+j q \varepsilon\left(1-e^{-j w \varepsilon}\right) \frac{\partial F_{2}^{(2)}(w, \varepsilon)}{\partial w}=0, \\
-e^{-j w \varepsilon} G_{1}(1+q) F_{0}^{(2)}(w, \varepsilon)+\left[\lambda\left(e^{j w \varepsilon}+1\right)+\gamma_{1}-G_{1}\left(q e^{-j w \varepsilon}-1\right)\right] F_{1}^{(2)}(w, \varepsilon)+ \\
\quad+\left(\lambda-G_{1} q e^{-j w \varepsilon}\right) F_{2}^{(2)}(w, \varepsilon)+j \varepsilon e^{-j w \varepsilon}(1+q) \frac{\partial F_{0}^{(2)}(w, \varepsilon)}{\partial w}+ \\
\quad+j \varepsilon\left(q e^{-j w \varepsilon}-1\right) \frac{\partial F_{1}^{(2)}(w, \varepsilon)}{\partial w}+j q \varepsilon e^{-j w \varepsilon} \frac{\partial F_{2}^{(2)}(w, \varepsilon)}{\partial w}=0
\end{array}\right.
$$

When $\varepsilon \rightarrow 0$ in $(24)$ and $\lim _{\varepsilon \rightarrow 0} F_{k}^{(2)}(w, \varepsilon)=F_{k}^{(2)}(w), k=\{0,1,2\}$ we obtain

$$
\left\{\begin{array}{l}
-\left(\lambda+\gamma_{0}+G_{1}\right) F_{0}^{(2)}(w)+\left(\mu+\lambda+G_{1}\right) F_{1}^{(2)}(w)+\gamma_{2} F_{2}^{(2)}(w)=0 \\
\left(\lambda+G_{1}\right) F_{0}^{(2)}(w)-\left(\lambda+\mu+\gamma_{1}+G_{1}\right) F_{1}^{(2)}(w)=0 \\
\gamma_{0} F_{0}^{(2)}(w)+\gamma_{1} F_{1}^{(2)}(w)-\gamma_{2} F_{2}^{(2)}(w)=0 \\
-G_{1}(1+q) F_{0}^{(2)}(w)+\left[2 \lambda+\gamma_{1}-G_{1}(q-1)\right] F_{1}^{(2)}(w)+\left(\lambda-G_{1} q\right) F_{2}^{(2)}(w)=0
\end{array}\right.
$$

The solution of systems of equations (24) has the following form

$$
\left\{\begin{array}{l}
F_{k}^{(2)}(w, \varepsilon)=\left(R_{k}+j w \varepsilon f_{k}\right) \Phi^{(2)}(w)+o\left(\varepsilon^{2}\right), \quad k=\{0,1,2\} \\
R_{0}+R_{1}+R_{2}=1
\end{array}\right.
$$

where $R_{0}, R_{1}, R_{2}$ are defined above, $f_{0}, f_{1}, f_{2}$ are constants and function $\Phi^{(2)}(w)$ is to be determined.

Substituting (26) into (24) and taking into account (25), with the proviso that $\varepsilon \rightarrow 0$ one can write the system as

$$
\left\{\begin{array}{l}
{\left[\left(\lambda+\gamma_{0}+G_{1}\right) f_{0}+G_{1} q R_{0}-\left(\mu+\lambda+G_{1}\right) f_{1}-\left(2 \lambda+G_{1}\right) R_{1}-\gamma_{2} f_{2}\right] w \Phi^{(2)}(w)=} \\
=\left(R_{0}-R_{1}\right) \frac{d \Phi^{(2)}(w)}{d w}, \\
{\left[\left(\lambda+G_{1}\right) f_{0}-G_{1} R_{0}-\left(\lambda+\mu+\gamma_{1}+G_{1}\right) f_{1}-G_{1} q R_{1}\right] w \Phi^{(2)}(w)=\left(R_{0}-R_{1}\right) \frac{d \Phi^{(2)}(w)}{d w},} \\
{\left[\gamma_{0} f_{0}+\gamma_{1} f_{1}+\gamma_{1} R_{1}-\gamma_{2} f_{2}-\left(G_{1} q-\lambda\right) R_{2}\right] w \Phi^{(2)}(w)=0,} \\
{\left[\left(2 \lambda+\gamma_{1}+G_{1}-G_{1} q\right) f_{1}+\left(\lambda+G_{1} q\right) R_{1}-\left(\lambda-G_{1} q\right) f_{2}+G_{1} q R_{2}-G_{1}(1+q) f_{0}+\right.} \\
\left.\quad+G_{1}(1+q) R_{0}\right] w \Phi^{(2)}(w)=\left[(1-q) R_{1}-(1+q) R_{0}-q R_{2}\right] \frac{d \Phi^{(2)}(w)}{d w},
\end{array}\right.
$$


where $R_{0}, R_{1}$ and $G_{1}$ are defined in (18), (19) and (16), respectively.

The solution of system (27) has the form

$$
\Phi^{(2)}(w)=\exp \left\{G_{2} \frac{(j w)^{2}}{2}\right\},
$$

where $G_{2}$ is defined in (5).

Using the same transformation as for the first-order asymptotic and additional conditions $f_{2}-f_{1}-f_{0}=0$ and $f_{1}-f_{0}=0$, we finally obtain expressions for the solution of system (27)

$$
\left\{\begin{array}{l}
\quad\left(\lambda+\gamma_{0}+G_{1}\right) f_{0}+G_{1} q R_{0}-\left(\mu+\lambda+G_{1}\right) f_{1}-\left(2 \lambda+G_{1}\right) R_{1}-\gamma_{2} f_{2}= \\
\quad=\left(\lambda+G_{1}\right) f_{0}-G_{1} R_{0}-\left(\lambda+\mu+\gamma_{1}+G_{1}\right) f_{1}-G_{1} q R_{1}, \\
\gamma_{0} f_{0}+\gamma_{1} f_{1}+\gamma_{1} R_{1}-\gamma_{2} f_{2}-\left(G_{1} q-\lambda\right) R_{2}=0, \\
2 f_{1}+f_{2}=0 \\
f_{1}-f_{0}=0 .
\end{array}\right.
$$

Making the reverse substitutions we obtain

$$
H_{k}^{(2)}(u)=F_{k}^{(2)}(w, \varepsilon)=\left(R_{k}+j w \varepsilon f_{k}\right) \exp \left\{G_{2} \frac{(j w)^{2}}{2}\right\}+o\left(\varepsilon^{2}\right) \approx R_{k} \exp \left\{\frac{G_{2}}{\sigma} \frac{(j u)^{2}}{2}\right\} .
$$

Then using (30), expressions (22) can be written as

$$
H_{k}(u)=\exp \left\{\frac{G_{1}}{\sigma} j u\right\} H_{k}^{(2)}(u) \approx R_{k} \exp \left\{\frac{G_{1}}{\sigma} j u+\frac{G_{2}}{\sigma} \frac{(j u)^{2}}{2}\right\}, \quad k=\{0,1,2\} .
$$

Taking into account (31), characteristic function $H(u)=H_{0}(u)+H_{1}(u)+H_{2}(u)$. Assuming that customers in the orbit have long delays and the "patience" is high, we can see that distribution is the Gaussian one. Hence

$$
h_{2}(u)=\exp \left\{\frac{G_{1}}{\sigma} j u+\frac{G_{2}}{\sigma} \frac{(j u)^{2}}{2}\right\} .
$$

Theorem 3.1 is proved.

\section{Numerical results}

In this section we give some comments to Theorem 3.1 and several numerical examples are considered.

We construct asymptotic distributions of the probabilities of the number of customers in the orbit with parameters $\mu=1, \gamma_{0}=0.1, \gamma_{1}=0.2, \gamma_{2}=1, \alpha=2 \sigma$ for various values of the delay parameter $\sigma$ and parameter $\lambda$. Then these distributions are compared with pre-limit (numerical) distributions obtained by the matrix method.

Fig. 2 shows one of samples for $\lambda=0.7$ and $\sigma=0.01$ (left picture) and $\sigma=0.001$ (right picture).

As a measure of proximity of two distributions the Kolmogorov distance

$$
\Delta=\max _{0 \leqslant i \leqslant N}\left|\sum_{k=0}^{i} P_{\text {matrix }}(i)-\sum_{k=0}^{i} P_{\text {asimpt }}(i)\right|
$$

is used, where $P_{\text {matrix }}(i)$ is the probability distribution of the number of customers in the orbit obtained by the matrix method, $P_{\text {asimpt }}(i)$ is the asymptotic probability distribution of the number of customers in the orbit. 

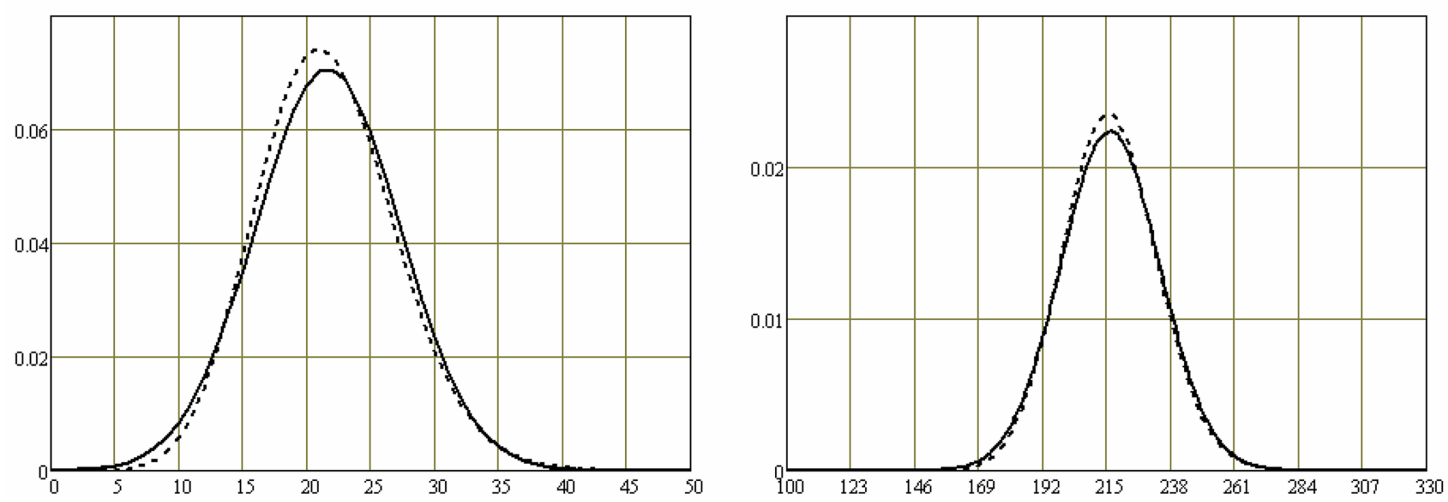

Fig. 2. Asymptotic (dashed line) and pre-limit(numerical) (solid line) probability distributions of the number of calls in the orbit

Table 1. Values of the Kolmogorov distance

\begin{tabular}{|c|c|c|c|c|c|}
\hline \multirow{2}{*}{$\lambda / \mu$} & \multicolumn{5}{|c|}{ Kolmogorov distance $\Delta$} \\
\cline { 2 - 6 } & $\sigma=0.1$ & $\sigma=0.05$ & $\sigma=0.01$ & $\sigma=0.005$ & $\sigma=0.001$ \\
\hline 0.5 & 0.161 & 0.101 & 0.023 & 0.016 & 0.009 \\
\hline 0.7 & 0.117 & 0.066 & 0.020 & 0.016 & 0.013 \\
\hline 0.9 & 0.092 & 0.048 & 0.021 & 0.020 & 0.018 \\
\hline 1.1 & 0.075 & 0.039 & 0.024 & 0.023 & 0.022 \\
\hline 1.5 & 0.055 & 0.035 & 0.030 & 0.029 & 0.029 \\
\hline 2.0 & 0.046 & 0.037 & 0.035 & 0.035 & 0.035 \\
\hline
\end{tabular}

\section{Conclusion}

Retrial queueing system of $M / M / 1$ type with impatient customers in the orbit, collisions and unreliable server is considered in the paper. It is proved that stationary probability distribution of the number of customers in the orbit can be approximated by the Gaussian distribution under conditions of a long delay and a long patience time of the customers in the orbit. The accuracy of the approximation was compared with numerical results obtained with the use of the matrix analytic method.

The study was funded by Russian Foundation for Basic Research and Tomsk region (project no. 19-41-703002).

\section{References}

[1] J.R.Artalejo, A.Gomez-Corral, Retrial Queueing Systems: A Computational Approach, Springer, 2008.

[2] J.R.Artalejo, G.I.Falin, Standard and retrial queueing systems: A comparative analysis, Revista Matematica Complutense, 15(2002), 101-129.

[3] G.I.Falin, J.G.C.Templeton, Retrial queues, London, New York: Chapman \& Hall, 1997. 
[4] E.Yu.Danilyuk, E.A.Fedorova, S.P.Moiseeva, Asymptotic Analysis of an Retrial Queueing System M/M/1 with Collisions and Impatient Calls, Automation and Remote Control, 79(2018), no. 12, 2136-2146. DOI: 10.1134/S0005117918120044.

[5] A.Nazarov, J.Sztrik, A.Kvach, T.Berczes, , Annals of Operations Research, 277(2019), no. 2, 213-229. DOI: 10.1007/s10479-018-2894-z.

[6] L.Lakaour, D.Aïssani, K.Adel-Aissanou, K.Barkaoui, Methodology and Computing in Applied Probability, 21(2019), no. 4, 1395-1406.

[7] E.Danilyuk, S.Moiseeva, A.Nazarov, Asymptotic Analysis of Retrial Queueing System M/GI/1 with Collisions and Impatient Calls, In: Dudin A., Nazarov A., Moiseev A. (eds). Information Technologies and Mathematical Modelling. Queueing Theory and Applications. ITMM 2019, Communications in Computer and Information Science, Vol. 1109, 2019, 230 242 (doi: 10.1007/978-3-030-33388-1_19).

[8] J.Kim, Communications of the Korean Mathematical Society, 4(2010), 647-653.

[9] O.Vygovskaya, E.Danilyuk, S.Moiseeva, In: Dudin A., Nazarov A., Moiseev A. (eds). Information Technologies and Mathematical Modelling. Queueing Theory and Applications. ITMM 2018, WRQ 2018. Communications in Computer and Information Science, Vol. 912, 2018, 387-399. DOI: 10.1007/978-3-319-97595-5_30.

[10] E.Danilyuk, O.Vygovskaya, S.Moiseeva, S.Rozhkova, In: Vishnevskiy V., Kozyrev D. (eds). Distributed Computer and Communication Networks. DCCN 2018. Communications in Computer and Information Science, Vol. 919, 2018, 493-504. DOI: 10.1007/978-3-319-99447$5 \_42$.

[11] E.Fedorova, E.Danilyuk, A.Nazarov, A.Melikov, In: Phung-Duc T., Kasahara S., Wittevrongel S. (eds) Queueing Theory and Network Applications. QTNA 2019. Lecture Notes in Computer Science, Vol. 11688, 2019, 3-15. DOI: 10.1007/978-3-030-27181-7_1.

[12] M.P.D'Arienzo, A.N.Dudin, S.A.Dudin, R.Manzo, Journal of Ambient Intelligence and Humanized Computing, (2019), 1-9.

[13] B.Kim, J.Kim, Statistics \& Probability Letters, 134(2018), 54-62.

[14] A.Aissani, F.Lounis, D.Hamadouche, S.Taleb, American Journal of Mathematical and Management Sciences, 38(2019), no. 2, 125-150.

[15] A.N.Dudin, Operations Research, 5(2018), 245-255.

[16] C.J.Ancker, A.V.Gafarian, 11(1963), no. 1, 88-100. DOI: 10.1287/opre.11.1.88.

[17] F.A.Haight, Biometrika, 44(1957), no. 3/4, 360-369. DOI: 10.2307/2332868.

[18] D.Y.Barrer, Operations Research, 5(1957), no. 5, 644-649. DOI: 10.1287/opre.5.5.644.

[19] R.Kumar, S.K.Sharma, International Journal of Mathematics in Operational Research, 5(2013), no. 6, 709-720. DOI: 10.1504/ijmor.2013.057488.

[20] R.Kumar, B.K.Som, Advance Modeling and optimization, 16(2014), no. 2, 339-353. 
[21] B.K.Som, Advances in Analytics and Applications, (2018), 261-272. DOI: 10.1007/978-98113-1208-3_20.

[22] A.Santhakumaran, B.Thangaraj, Information and Management Sciences, 11(2000), no. 3, $71-79$.

[23] B.K.Som, Reliability: Theory and Applications, 14(2019), no. 1(52), 87-101.

[24] K.Wang, N.Li, Z.Jiang, Proceedings of 2010 IEEE International Conference on Service Operations and Logistics, and Informatics, 2010, 82-87. DOI: 10.1109/SOLI.2010.5551611.

[25] A.Nazarov, J.Sztrik, A.Kvach, In: Dudin,A., Nazarov, A. (eds.). Information Technologies and Mathematical Modelling. Queueing Theory and Applications. ITMM 2017, CCIS, Vol. 800, 2017, 97-110. DOI: 10.1007/978-3-319-68069-9.

[26] T.Berczes, J.Sztrik, A.Toth, A.Nazarov, In: Dudin,A., Nazarov, A. (eds.). Information Technologies and Mathematical Modelling. Queueing Theory and Applications. ITMM 2017, CCIS, Vol. 800, 2017, 248-258. DOI: 10.1007/978-3-319-68069-9.

\title{
Асимптотический анализ системы массового обслуживания с повторными вызовами $\mathrm{M} / \mathrm{M} / 1$ с нетерпеливыми заявками, конфликтами и ненадежным прибором
}

Елена Ю. Данилюк

Светлана П. Моисеева

Национальный исследовательский Томский государственный университет Томск, Российская Федерация

Янош Стрик

Университет Дебрецена

Дебрецен, Венгрия

\begin{abstract}
Аннотация. В настоящей статье мы рассматриваем систему массового обслуживания с повторными вызовами (RQ-систему) типа M/M/1 с пуассоновским потоком поступающих в систему заявок и одним сервером, обслуживание которым имеет экспоненциальное распределение. Классическая модель RQ-системы усложнена наличием конфликтов заявок в системе, "нетерпеливых" заявок на орбите, а также "ненадежным" прибором, который выходит из строя и ремонтируется в функционирующей системе массового обслуживания. Время, через которое заявки с орбиты вновь обращаются к обслуживающему прибору; время, через которое заявки с орбиты покидают систему, время, в течение которого сервер находится в рабочем состоянии (в зависимости от того, занят прибор обслуживанием заявки или нет, а также время, в течение которого длится ремонт вышедшего из строя сервера, распределены экспоненциально. Мы используем метод асимптотического анализа для решения задачи нахождения распределения вероятностей числа заявок на орбите. В качестве асимптотического условия предлагается условие высокой загрузки системы и долгой "терпеливости" заявок на орбите. Формулируется и доказывается теорема об асимптотически гауссовском распределении вероятностей числа заявок на орбите. Приводятся численные результаты, демонстрирующие область применения полученных теоретических выводов.
\end{abstract}

Ключевые слова: RQ-система, нетерпеливые заявки, конфликты, ненадежный прибор, асимптотический анализ. 